\title{
Alternatives to standard acute in-patient care in England: differences in content of care and staff-patient contact
}

B. Lloyd-Evans, S. Johnson, N. Morant, H. Gilburt, D. P. J. Osborn, D. Jagielska, R. Skinner, M. Leese, G. Shepherd and M. Slade

\section{Background}

Differences in the content of care provided by acute in-patient mental health wards and residential crisis services such as crisis houses have not been researched.

\begin{abstract}
Aims
To compare planned and actual care provided at alternative and standard acute wards and to investigate the relationship between care received and patient satisfaction.
\end{abstract}

\section{Method}

Perspectives of stakeholders, including local service managers, clinicians and commissioners, were obtained from 23 qualitative interviews. Quantitative investigation of the care provided at four alternative and four standard services was undertaken using three instruments developed for this study. The relationship of care received to patient satisfaction was explored.

\section{Results}

No significant difference was found in intensity of staffpatient contact between alternative and standard services. Alternative services provided more psychological and less physical and pharmacological care than standard wards. Care provision may be more collaborative and informal in alternative services. All measured types of care were positively associated with patient satisfaction. Measured differences in the care provided did not explain the greater acceptability of community alternatives.

\section{Conclusions}

Similarities in care may be more marked than differences at alternative and standard services. Staff-patient contact is an important determinant of patient satisfaction, so increasing it should be a priority for all acute in-patient services.

\section{Declaration of interest}

None.

about the nature of care provided by alternative services and whether this differed from that provided in acute wards. A topic guide developed for the interviews covered the role of the alternative service, pathways to care and the model and content of care provided. Participants were also asked to comment on quantitative data regarding characteristics and outcomes of patients at the local alternative and standard services. ${ }^{14,15}$

\section{Measures}

Service content was measured using three instruments, the Camden Staff-Patient Activity Record (CaSPAR), the Camden Record of Inpatient Care Events (CaRICE) and the Camden Content of Care Questionnaire - Patient version (CCCQ-P), developed for this study. The instruments and their properties are fully described elsewhere. ${ }^{16}$ CaSPAR uses direct observation and staff report to measure the number and proportion of patients in contact with staff at predefined time points. CaRICE aggregates information provided daily by all staff to calculate the time in minutes of staff contact provided per patient. CCCQ-P is a patient-completed questionnaire about the types and frequency of care received during an admission. CaRICE and CCCQ-P categorise care into 21 types, from which subscale scores can be derived for provision of social, psychological and physical and pharmacological interventions. The categories of care used in CaRICE and CCCQ-P are listed in Table 1. Patient satisfaction was assessed using the Client Satisfaction Questionnaire (CSQ), an eight-item self-report measure. ${ }^{17}$

\section{Settings}

Data were collected from four alternative and four local comparison standard in-patient services, a subset of those
Semi-structured interviews were used to obtain the views of stakeholders (key individuals within the local acute care system) 
described by Johnson et al. ${ }^{14}$ The four alternatives were three community-based services - crisis team beds, a clinical crisis house and a non-clinical alternative (a crisis house for Black and minority ethnic service users) - and an in-patient ward using the Tidal Model, a nursing-led model designed to enhance collaboration and contact between in-patient staff and patients. ${ }^{18}$

\section{Procedures}

Twenty-three semi-structured interviews were conducted with stakeholders of the alternative services, including at each service the alternative service manager, representatives of the local standard in-patient service, the management of the local mental health trust and the commissioning agency, the manager from the local crisis and home treatment team, and a consultant psychiatrist from a community service referring patients to the alternative service. At the crisis team beds service, the alternative service manager was also the local crisis team manager, so one fewer interview was conducted than at other services. Interviews were recorded and transcribed verbatim.

Twenty-eight CaSPAR recordings were made by researchers at each service at preset time points (four per day) and including weekend and evening recordings. All staff were asked to complete CaRICE over a 5-day (Monday to Friday) recording period at each service, describing the interventions they had delivered during each working shift. Researchers were present during recording periods to distribute and collect forms. The CCCQ-P and CSQ were completed as a structured interview with patients at or close to their discharge from the service, describing the interventions they had received during the admission as a whole. Written informed consent was obtained from participants, following an initial approach from service staff. Researchers sought to recruit 40 participants at each service. All quantitative data were entered on an electronic database using SPSS for Windows, version 14.

\section{Statistical analysis}

Thematic content analysis was used to analyse data from stakeholder interviews, corresponding broadly to the procedures described by Coffey \& Atkinson. ${ }^{19}$ A coding frame was developed collaboratively by two authors (B.L.E. and N.M.) and main themes explored using QSR Nvivo7 software (www.qsrinternational.com). The quantitative analyses of care provided at alternative and standard services compared the three community-based alternatives and their local comparison standard services. Data from the Tidal Model ward and its comparison service were excluded from main analyses. Its role was as a local standard acute ward. ${ }^{14}$ Stakeholders reported inadequate implementation of the model and that the resulting service did not offer an alternative to standard acute care. Descriptive data from the two excluded services are presented separately.

Linear regression was used to test whether community alternatives and standard services differed in intensity of staff-patient contact and types of care provided, measured by CaSPAR and CCCQ-P. The CaSPAR data were analysed adjusting for time and day of recordings and clustering by service. The CCCQ-P data were analysed adjusting for patient characteristics - Mental Health Act status (detained or voluntary), gender, age and ethnicity (Black, White, Asian or other) - and clustering by service. The limited power of data from CaRICE, which yields aggregate data for the types and intensity of care provided at a service as a whole, meant that clinically important differences between alternative and standard services were unlikely to achieve statistical significance. We therefore assessed not only whether differences between service types were statistically significant, but also the effect size (the difference in mean scores divided by the standard deviation of all data). Following standard advice on estimating effect size, our assumption was that a score of $0.2-0.5$ indicates a small effect, $0.5-0.8$ a medium effect and over 0.8 a large effect. ${ }^{20}$

A model of patient satisfaction with residential acute care was explored using regression analyses. The relationship between patient satisfaction and service type was analysed, adjusting for patient characteristics and clustering by service. Additional adjustment for CCCQ-P variables was then made, to explore how care received relates to satisfaction and whether adjusting for types of care received affects the relationship between satisfaction and service type. The CCCQ-P data from all eight services were included in this model of satisfaction. The Tidal Model ward was included as a standard in-patient service in these explorations of the relationship between service type and patient satisfaction, so this part of the analysis involved comparing three community crisis houses with five acute in-patient wards.

\section{Results}

\section{Stakeholder interviews}

A common perception was that alternative services provide more staff time and attention to patients than acute wards: 11 out of 23 interviewees made reference to this, including the service managers of all four alternatives. The Tidal Model was perceived by stakeholders as having been initially received positively by patients, increasing one-to-one staff contact with patients and facilitating collaborative agenda-setting in ward rounds. An erosion of its model fidelity and implementation was identified, however. Fatigue from the simultaneous implementation of too many different initiatives, lack of active management support, and resistance to change and to proactive patient engagement among nurses were identified as barriers to its sustained use, resulting in the model having minimal sustained impact on actual service delivery.

Stakeholders commenting on community alternatives did not identify explicit models of care being used. Types of intervention available were not felt to differ greatly from acute wards. Most commonly highlighted was the inability of community alternative services to provide $24 \mathrm{~h}$ medical support and lack of facilities to provide complex physical healthcare. In-patient service representatives suggested that greater service size and the skills of a professionally trained and eclectic staff group resulted in a greater range of care being available on acute wards. However, particularly for the clinical crisis house and crisis team beds, similarities in care to acute wards were emphasised as much as differences. Additional interventions were not consistently described as available in alternative services; rather, a distinctive style of care was proposed by stakeholders as characteristic of these services. Care was seen as typically more individual, collaborative and informal than on acute wards, resulting in patients experiencing greater choice and control during their stay. Excerpts from interview transcripts illustrating themes from analysis of stakeholder interviews are provided in the online supplement to this paper.

\section{Quantitative comparison of care}

Twenty-eight CaSPAR recordings were made at each service. The status (with staff or not) was identified for $99 \%$ of patients resident at recording times. Five days of CaRICE data were collected from each service. Completed forms were obtained from $871(94.7 \%)$ of 919 eligible staff. Completed CCCQ-P and CSQ questionnaires were obtained from $314(70.2 \%)$ of 447 patients who were approached to participate in the study. Missing data from CCCQ-P forms required the exclusion of up to 11 CCCQ-P responses from analyses. 
Table 1 Content of care at three alternative and three standard services

\begin{tabular}{|c|c|c|c|c|}
\hline & \multicolumn{4}{|c|}{ Score: mean (s.d.) } \\
\hline & $\begin{array}{l}\text { Community } \\
\text { alternatives }\end{array}$ & $\begin{array}{l}\text { Standard } \\
\text { services }\end{array}$ & $\begin{array}{l}\text { Tidal Model } \\
\text { ward }\end{array}$ & $\begin{array}{c}\text { Tidal Model } \\
\text { comparison service }\end{array}$ \\
\hline \multicolumn{5}{|l|}{ Total care (21 categories) ${ }^{a}$} \\
\hline \multicolumn{5}{|l|}{ CaSPAR } \\
\hline Proportion of patients with staff, \% (28 recordings per service) & $12.3(20.9)$ & $11.8(9.4)$ & $10.8(10.3)$ & $8.8(8.0)$ \\
\hline \multicolumn{5}{|l|}{ CaRICE } \\
\hline Contact per patient per day, min (5 days' data per service) & $149.6(39.1)$ & $135.9(23.1)$ & $109.6(26.2)$ & $82.6(5.6)$ \\
\hline CCCQ-P total care (range 0-147) $(n=217)$ & $25.7(16.2)$ & $30.6(15.5)$ & $31.9(15.3)$ & $34.4(18.9)$ \\
\hline \multicolumn{5}{|l|}{ Social interventions (7 categories) ${ }^{b}$} \\
\hline CaRICE & $60.7(35.6)$ & $43.8(17.7)$ & $43.3(7.0)$ & $32.6(7.3)$ \\
\hline CCCQ-P subscale (range 0-49) $(n=222)$ & $7.4(6.6)$ & $7.9(6.4)$ & $8.7(6.7)$ & $7.6(7.2)$ \\
\hline \multicolumn{5}{|l|}{ 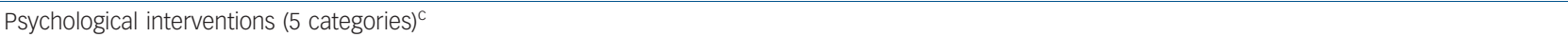 } \\
\hline CaRICE & $29.7(24.6)$ & $19.8(5.7)$ & $18.4(10.5)$ & $16.4(2.2)$ \\
\hline CCCQ-P subscale (range 0-35) $(n=221)$ & $5.7(6.3)$ & $4.7(5.8)$ & $4.8(5.5)$ & $6.6(6.9)$ \\
\hline \multicolumn{5}{|l|}{ Physical and pharmacological interventions (6 categories) ${ }^{d}$} \\
\hline CaRICE & $31.9(30.0)$ & $48.3(16.6)$ & $28.8(15.4)$ & $20.4(6.4)$ \\
\hline CCCQ-P subscale (range 0-42) $(n=220)$ & $7.7(5.8)$ & $12.7(5.8)$ & $12.5(3.9)$ & $14.1(5.7)$ \\
\hline \multicolumn{5}{|c|}{$\begin{array}{l}\text { CaRICE, Camden Staff-Patient Activity Record; CCCQ-P, Camden Content of Care Questionnaire - Patient version. } \\
\text { a. Categories as listed in subscales below plus assessment, care planning meetings, aftercare. } \\
\text { b. Categories are housing, finances, legal issues, current activity, future activity, activities of daily living, family support. } \\
\text { c. Categories are help with past events, current relationships, drugs and alcohol, symptom coping and illness education. } \\
\text { d. Categories are medication review, concordance, practical help, physical healthcare, observations, restraint. }\end{array}$} \\
\hline
\end{tabular}

Table 1 provides descriptive data from CaSPAR, CaRICE and CCCQ-P. Data from three community-based alternatives and from their comparison services are aggregated; results from the Tidal Model ward and its comparison service are presented separately. Scores for the Tidal Model ward were more similar to mean scores for standard in-patient wards than to mean scores for community alternatives on total care variables from all three measures and five out of six subscale variables. Results indicate that the intensity of contact and types of care provided at the Tidal Model ward at the time of recording were broadly similar to standard wards.

Statistical comparison of CaSPAR and CCCQ-P scores from community alternative services and standard wards is provided in Table 2, reporting results from regression analyses. No significant difference in intensity of staff-patient contact between alternative and standard services was identified by either measure. The CCCQ-P subscale data indicate significantly greater provision of psychological interventions and less provision of physical and pharmacological interventions at the alternative services, but no significant difference in social interventions.

Analyses and estimates of effect size for community alternatives compared with standard services from CaRICE data are presented in Table 3. No significant difference in results was found between groups but the limited power of analyses using CaRICE data must be noted. Wide confidence intervals from $t$-tests reported in Table 3 indicate the possibility of clinically important differences in care between alternative and standard services. Examination of effect sizes suggests, consistent with CCCQ-P results, only a small difference between groups in intensity of care provided and a medium effect for alternative services of more psychological care and less physical and pharmacological care. A medium effect size was also found for more social care at alternative services: this indicates that the

\begin{tabular}{|c|c|c|c|}
\hline & $R^{2}$ & Correlation coefficient $(95 \% \mathrm{Cl})^{\mathrm{a}}$ & $P$ \\
\hline \multicolumn{4}{|l|}{ CASPAR } \\
\hline \multicolumn{4}{|l|}{ Total score } \\
\hline Model $1^{\mathrm{b}}$ & $<0.01$ & $-0.45(-12.68$ to 11.78$)$ & 0.93 \\
\hline Model $2^{c}$ & 0.03 & $-0.45(-12.98$ to 12.03$)$ & 0.93 \\
\hline \multicolumn{4}{|l|}{ CCCQ-P } \\
\hline \multicolumn{4}{|c|}{ Total score $(n=217)$} \\
\hline Model 1 & 0.02 & $4.85(-2.70$ to 12.41$)$ & 0.16 \\
\hline Model 2 & 0.06 & $3.31(-2.27$ to 8.90$)$ & 0.19 \\
\hline \multicolumn{4}{|c|}{ Social interventions subscale score $(n=222)$} \\
\hline Model 1 & $<0.01$ & $0.49(-1.00$ to 1.97$)$ & 0.44 \\
\hline Model 2 & 0.03 & $0.02(-2.09$ to 2.14$)$ & 0.98 \\
\hline \multicolumn{4}{|c|}{ Psychological interventions subscale score $(n=221)$} \\
\hline Model 1 & 0.01 & $-0.97(-2.80$ to 0.85$)$ & 0.23 \\
\hline Model 2 & 0.04 & $-1.33(-2.48$ to -0.18$)$ & $0.03^{*}$ \\
\hline \multicolumn{4}{|c|}{ Physical and pharmacological interventions subscale score $(n=221)$} \\
\hline Model 1 & 0.16 & $5.03(-1.01$ to 11.08$)$ & 0.09 \\
\hline Model 2 & 0.25 & 4.35 (0.75 to 7.96$)$ & $0.03^{*}$ \\
\hline \multicolumn{4}{|c|}{$\begin{array}{l}\text { CaRICE, Camden Staff-Patient Activity Record; CCCQ-P, Camden Content of Care Questionnaire - Patient version. } \\
\text { a. Negative correlation coefficient indicates higher score at alternative services. } \\
\text { b. Model 1: relationship to service type (alternative } v \text {. standard service). } \\
\text { C. Model 2: relationship to service type, adjusting for patients' Mental Health Act status at admission, age, gender and ethnicity. } \\
{ }^{*} P<0.05 \text {. }\end{array}$} \\
\hline
\end{tabular}




\begin{tabular}{|c|c|c|c|c|c|}
\hline CaRICE domain & $\begin{array}{c}\text { Contact per patient per day, } \min \\
\text { Mean difference }{ }^{a}\end{array}$ & $95 \% \mathrm{Cl}$ & $t$ & $P$ & $\begin{array}{c}\text { Estimate } \\
\text { of effect size }\end{array}$ \\
\hline Total care score & 13.7 & -10.6 to 38.0 & 1.17 & 0.26 & 0.42 (small) \\
\hline Social care & 17.0 & -4.4 to 38.3 & 1.66 & 0.11 & 0.59 (medium) \\
\hline Psychological care & 9.9 & -3.9 to 23.7 & 1.52 & 0.15 & 0.54 (medium) \\
\hline Physical and pharmacological care & -16.4 & -34.8 to 2.0 & -1.85 & 0.08 & 0.65 (medium) \\
\hline
\end{tabular}

duration of social interventions may be greater than at standard wards; CCCQ-P data indicated that social interventions were not delivered more often or more varied in type.

Descriptive data from content of care measures from individual services are provided in online Table DS1. Despite some divergence of service scores across measures, marked features of services' care provision can be consistently identified. The nonclinical crisis house, which employed no medical or nursing staff, ranked lowest of all services on both CaRICE and CCCQ-P for physical and pharmacological interventions. The crisis team beds scored lowest of all services on CaRICE and CCCQ-P for social interventions but highly on both measures for psychological interventions. A lack of emphasis on social problems may be explained by the brief length of stay at the crisis team beds service, typically less than 1 week. The clinical crisis house, which provides a daily structured programme of activities within the residential unit, scored highest on CaSPAR total score and CaRICE and CCCQ-P for the current activity category.

\section{Impact of care received on patient satisfaction}

Results of a model of patient satisfaction using regression analysis including CSQ and CCCQ-P data are presented in Table 4. All CCCQ-P subscale scores were significantly positively associated with patient satisfaction, with more of each type of care linked to greater satisfaction. The $R^{2}$ values indicate that variance in CSQ scores could be explained more by analysis including CCCQ-P total care score than any subscale score. The amount of care received by patients may therefore be more influential on their satisfaction with services than the broad types of care received. Patient satisfaction with community alternatives remained significantly greater than at standard wards after adjustment for all CCCQ-P variables, suggesting that differences in the amount of care or types of intervention provided might not be primary influences on patients' greater satisfaction with alternatives. The $R^{2}$ values indicate that nearly three-quarters of the variance in patient satisfaction could not be explained by variables included in this model. The biggest change to the correlation coefficient for service type followed adjustment for CCCQ-P physical and pharmacological interventions: the increase in correlation coefficient following adjustment suggests that community alternatives are more acceptable than standard wards despite, not because of, providing less physical and pharmacological care.

\section{Discussion}

Alternative services were commonly perceived by stakeholders as giving more staff time to patients than standard in-patient

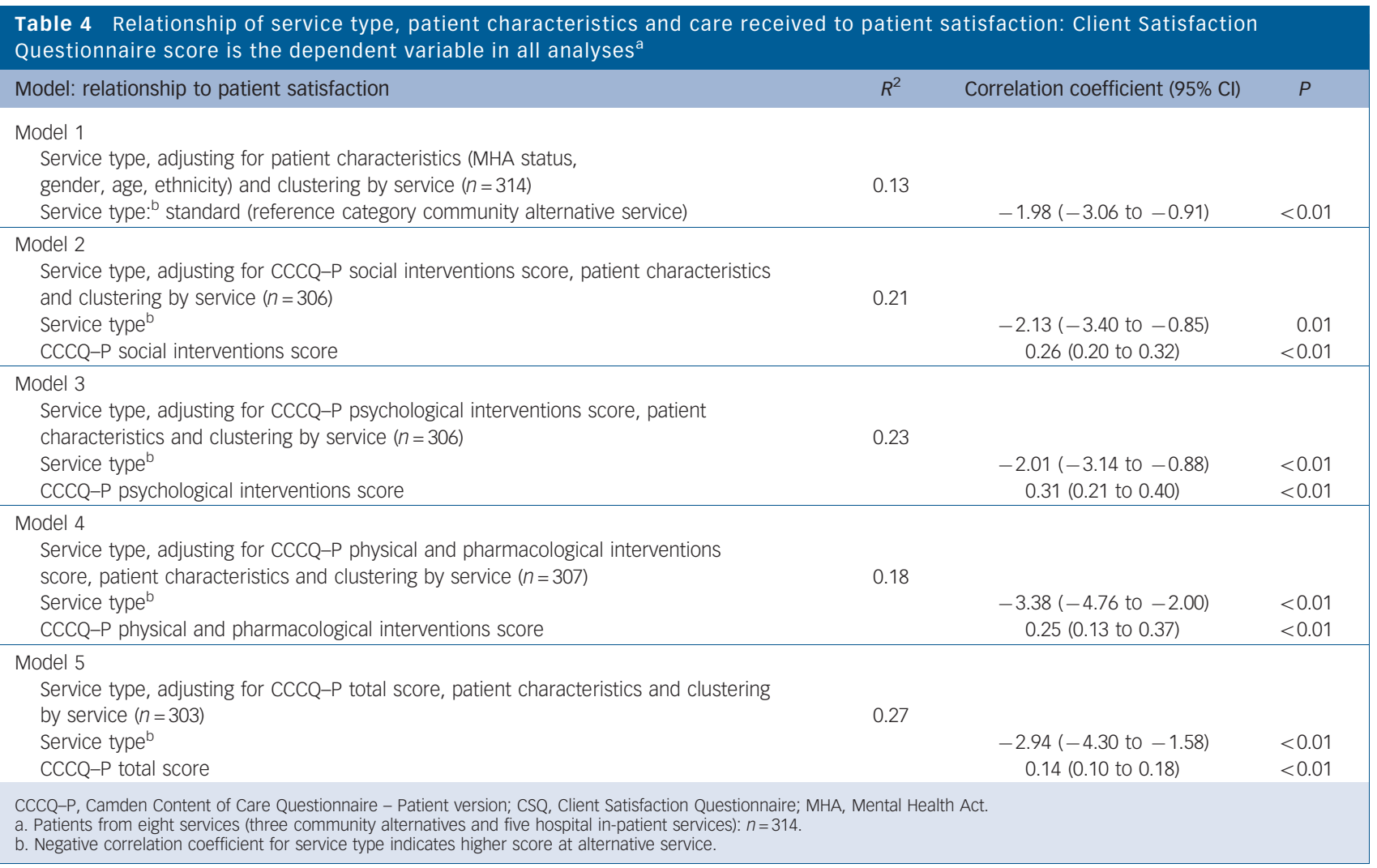


services. Other than greater access to medical care on acute wards, few differences in interventions provided or models of care were identified. Care in community alternatives was seen as more collaborative and informal than at standard wards. Multiple method quantitative assessment found no significant difference in the intensity of staff-patient contact at alternative and standard services. There was greater provision of psychological care at community alternatives and of physical and pharmacological care at standard wards. No evidence was found that care at the Tidal Model ward differed from standard in-patient care. There was preliminary evidence of differences in care provision between different types of community alternatives. No broad type of care was experienced on average as aversive by patients. The amount of direct care received may be more important than the type of intervention in explaining patient satisfaction, although neither has a substantial role in explaining greater patient satisfaction at alternative services than at acute wards.

\section{Limitations}

This study has three main limitations. First, only a small number of patient characteristics could be adjusted for in comparisons of alternative and standard services. This limits understanding of how far differences in care reflect different needs and presentations of the patients admitted, or represent intrinsic differences in what services provide. Second, the study involves only one exemplar of each type of alternative. Evidence about care provision at different alternative service types is therefore preliminary. Third, limited depth of information is available about care at services. Although CaRICE and CCCQ-P measure 21 types of care, information about delivery of specific interventions, or the style or quality of care, is not provided and forms of interventions for which there is a clear evidence base will be grouped together with others for which the evidence is limited.

The measures of content of care developed for this study are methodologically innovative, overcoming a lack of existing measures appropriate for use in in-patient services. ${ }^{21}$ Further research has been proposed to build on the development and psychometric exploration of these measures that has already been undertaken, ${ }^{16}$ and to refine methods to investigate the care provided by in-patient mental health services. Given current knowledge about how to measure content of care in in-patient mental health services, the multiple method approach used in this study is appropriate. Triangulation of findings from more than one quantitative measure and qualitative data from patients and stakeholders can corroborate findings regarding similarities and differences in service provision at alternative and standard services. $^{22}$

\section{Clinical implications}

There was little evidence that differences in types of care provided at community alternatives and standard services are of great clinical importance. The CCCQ-P data showed significantly greater provision of (broadly defined) psychological interventions by alternative services, but the CaRICE data indicated that this amounted to less than 10 min more per patient per day. Given the known poor physical health of mental health service users, ${ }^{23}$ the provision of less physical healthcare in alternative services is of concern. Such services should ensure that their patients have proper access to community physical healthcare and screening that would be more routinely available in hospital settings. Since acute physical conditions may exacerbate or even cause mental health crises, alternative services also need mechanisms for ensuring patients receive a physical health check close to the point of admission. However, CCCQ-P item scores indicated that in all services except the non-clinical crisis house, patients typically received core psychiatric interventions such as medication prescription and provision. Lower levels of pharmacological interventions or safety measures such as observations might be appropriate for the client group at alternative services, who are less likely to be detained and more likely to be known to services, ${ }^{14}$ with a probable existing treatment plan. Stakeholders reported that shortfalls in pharmacological interventions at alternative services compared with acute wards might be mitigated by close collaboration with community services such as crisis teams. The conclusion that there is much similarity in care provision between alternative and standard wards is supported by stakeholder interviews and the lack of differences in service interventions identified by patients in qualitative interviews. ${ }^{22}$ Although stakeholder interviews suggested potentially important differences from standard wards in the style of service provision at alternatives, service planners and referrers should not conclude that alternatives offer fundamentally different types of care from standard wards. They may conclude that alternatives can form part of mainstream acute care provision.

The model of satisfaction presented in this paper does not support wholesale change in the types of care provided by acute residential services, nor a highly critical appraisal of care provision on standard acute wards. All broad types of care were positively received by patients. Reduced provision of medical-type interventions at alternatives was not found to be related to their greater acceptability to patients. This study suggests that a focus on increasing the amount of contact and care provided to patients should be a priority for clinicians and managers, above changing the types of interventions available.

Neither the Tidal Model nor community crisis houses were found to address an expressed concern of patients, ${ }^{6-8}$ and expectation of stakeholders, that the amount of staff contact available to patients needs to be increased. Interventions with a more specific focus and mechanism for increasing staff-patient contact may be required. Protected engagement time - when for set periods staff are relieved of administrative duties, the ward is closed to visitors and the office closed to staff - is one, as yet unevaluated, example of an attempt to achieve this. ${ }^{24}$ The daily activity programme at the clinical crisis house contributed to its high CaRICE and CCCQ-P item scores for current activity and top-ranking CaSPAR score for proportion of patients interacting with staff. Service managers should consider greater use of structured groups and recreational activities at services as a means to increase staff-patient contact.

\section{Research implications}

Nearly three-quarters of variance in patient satisfaction was unexplained by the model presented here. Stakeholders indicated that the nature of care may be more individual, consensual and informal at alternative services than on acute wards. This finding is corroborated by qualitative interviews with service users, who experienced less coercion and paternalistic care at alternative units, ${ }^{22}$ and to an extent by Ward Atmosphere Scale data ${ }^{25}$ which, before adjustment for patient variables, showed greater autonomy and support at alternative than standard services. The way in which things are done, rather than what is done, may be distinctive and important to patient experience at alternative services. Evaluation focusing on comparisons of service style or therapeutic alliance at alternative and standard wards may identify differences in care not measured by this study.

The lack of impact of the Tidal Model found in this study may be due to its inadequate implementation. Stakeholders from the 
Tidal Model ward recounted the difficulties in implementing innovation and changing culture on acute wards. A previous launch of the model within the trust had also been discontinued. Evaluations of the Tidal Model have typically involved newly implemented services and have been small-scale and of short duration. ${ }^{26-29}$ Research to define and measure fidelity to the Tidal Model, then evaluation of services where it is well established, would be useful.

Differences in care provision between the community alternatives in this study support the typology of alternative services developed from a national UK survey. ${ }^{13}$ Further investigation could establish whether there are consistent differences in service provision between types of alternative. This could inform the development and subsequent evaluation of models of acute residential care, assisting the goal of providing effective, acceptable services.

Brynmor Lloyd-Evans, PhD, Sonia Johnson, DM, Department of Mental Health Sciences, University College London; Nicola Morant, PhD, Department of Social and Developmental Psychology, University of Cambridge; Helen Gilburt, PhD, Health Service and Population Research Department, Institute of Psychiatry, King's College London; David P. J. Osborn, PhD, Dorota Jagielska, MA, Department of Mental Health Sciences, University College London; Rachel Skinner, MA, Morven Leese, P. J. Osbere, King's College London; Geoff Shepherd, PhD, Sainsbury Centre for Mental Health, London; Mike Slade, PhD, Health Service and Population Research Department, Institute of Psychiatry, King's College London, London, UK

Correspondence: Dr Brynmor Lloyd-Evans, Department of Mental Health Sciences, Charles Bell House, 67-73 Riding House Street, London W1W 7EJ, UK. Email: b.lloyd-evans@ucl.ac.uk

\section{Funding}

This project was funded by the National Institute for Health Research Service Delivery and Organisation programme (project number 08/1304/075).

\section{Acknowledgements}

This study was undertaken in the context of the NIHR Specialist Mental Health Biomedica Research Centre at the Institute of Psychiatry, King's College London and the South London and Maudsley NHS Foundation Trust. The study was supported by the Mental Health and Maudsley NHS Foundation Trust. The study was supported by the Mental Health
Research Network (MHRN) and associated with the MHRN acute care group (conveno S.J.). The views expressed in this paper are those of the authors and not necessarily those of the NHS, the NIHR or the Department of Health.

\section{References}

1 Department of Health. Mental Health Policy Implementation Guide: Adult Acute Inpatient Care Provision. Department of Health, 2005.

2 Muijen M. Acute hospital care: ineffective, inefficient and poorly organised. Psychiatr Bull 1999; 23: 257-9.

3 Quirk A, Lelliott $P$. What do we know about life on acute psychiatric wards in the UK? A review of the research evidence. Soc Sci Med 2001; 53: 1565-74.

4 Sainsbury Centre for Mental Health. Acute Problems: A Survey of the Quality of Care in Acute Psychiatric Wards. Sainsbury Centre, 1998.

5 Higgins R, Hurst K, Wistow G. Nursing acute psychiatric patients: a quantitative and qualitative study. J Adv Nurs 1999; 29: 52-63.

6 Baker S. Environmentally Friendly? Patients' Views of Conditions on Psychiatric Wards. Mind, 2000.

7 Rose D. Users' Voices: The Perspectives of Mental Health Service Users on Community and Hospital Care. Sainsbury Centre for Mental Health, 2001.
8 Sainsbury Centre for Mental Health. Search for Acute Solutions: Improving the Quality of Care in Acute Psychiatric Wards. SCMH, 2006.

9 Gilburt H, Rose D, Slade M. The importance of relationships in mental health care: a qualitative study of service users' experiences of psychiatric hospital admission in the UK. BMC Health Serv Res 2008; 8: 92.

10 Garcia I, Kennett C, Quraishi M, Durcan G. Acute Care 2004: A National Survey of Adult Psychiatric Wards in England. Sainsbury Centre for Mental Health, 2005.

11 Ford R, Durcan G, Warner L, Harvey P, Muijen M. One day survey by the Mental Health Act Commission of acute adult psychiatric wards in England and Wales. BMJ 1998; 317: 1279-83.

12 Lloyd-Evans B, Slade M, Jagielska D, Johnson S. Residential alternatives to acute psychiatric hospital admission: systematic review. Br J Psychiatry 2009; 195: 109-117.

13 Johnson S, Gilburt H, Lloyd-Evans B, Osborn DPJ, Boardman J, Leese M, et al. In-patient and residential alternatives to standard acute psychiatric wards in England. Br J Psychiatry 2009; 194: 456-63.

14 Johnson S, Lloyd-Evans B, Morant N, Gilburt H, Shepherd G, Slade M, et al. Alternatives to standard acute in-patient care in England: roles and populations served. Br J Psychiatry 2010 (suppl 53): s6-13.

15 Slade M, Byford S, Barrett B, Lloyd-Evans B, Gilburt H, Osborn DPJ, et al. Alternatives to standard acute in-patient care in England: short-term clinical outcomes and cost-effectiveness. Br J Psychiatry 2010 (suppl 53): s14-9.

16 Lloyd-Evans B, Slade M, Osborn DP, Skinner R, Johnson S. Developing and comparing methods for measuring the content of care in mental health services. Soc Psychiatry Psychiatr Epidemiol 2010; advance online publication: doi 10.1007/s-00127-007-0216-X.

17 Attkisson C, Zwick R. The Client Satisfaction Questionnaire: psychometric properties and correlations with service utilization and psychotherapy outcome. Eval Program Plann 1982; 5: 233-7.

18 Barker P. The Tidal Model: developing an empowering, person-centred approach to recovery within psychiatric and mental health nursing. J Psychiatr Ment Health Nurs 2001; 8: 233-40.

19 Coffey A, Atkinson P. Making Sense of Qualitative Data: Complementary Research Strategies: 26-53. Sage, 1996.

20 Cohen J. Statistical Power Analysis for the Behavioural Sciences (2nd edn) Erlbaum, 1988.

21 Lloyd-Evans B, Slade M, Johnson S. Assessing the content of mental health services: a review of measures. Soc Psychiatry Psychiatr Epidemiol 2007; 42: 673-82.

22 Gilburt H, Slade M, Rose D, Lloyd-Evans B, Johnson S, Osborn DPJ, et al. Service users' experiences of residential alternatives to standard acute wards: qualitative study of similarities and differences. Br J Psychiatry 2010 (suppl 53): s26-31.

23 Sokal J, Messias E, Dikerson F, Keyenbuhl J, Brown C, Goldberg P, et al. Comorbidity of medical illnesses among adults with serious mental illness who are receiving community psychiatric services. J Nerv Ment Dis 2004; 192: $421-7$.

24 Department of Health. From Values to Action: The Chief Nursing Officer's Review of Mental Health Nursing. Department of Health, 2006.

25 Osborn DPJ, Lloyd-Evans B, Johnson S, Gilburt H, Byford S, Leese M, et al. Residential alternatives to acute in-patient care in England: satisfaction, ward atmosphere and service user experiences. Br J Psychiatry 2010 (suppl 53): s41-5.

26 Stevenson C, Barker $\mathrm{P}$, Fletcher E. Judgement days: developing an evaluation for an innovative nursing model. J Psychiatr Ment Health Nurs 2002; 9 : 271-6.

27 Gordon W, Morton T, Brooks G. Launching the Tidal Model: evaluating the evidence. J Psychiatr Ment Health Nurs 2005; 12: 703-12.

28 Berger JL. Incorporation of the Tidal Model into the interdisciplinary plan of care - a program quality improvement project. J Psychiatr Ment Health Nurs 2006; 13: 464-7.

29 Lafferty S, Davidson R. Putting the person first. Ment Health Today 2006; Mar: $31-3$ 